\title{
Host-Enhanced Phenyl-Perfluorophenyl Polar $-\pi$ Interactions
}

\author{
Zehuan Huang, Xiaoyi Chen, Guanglu Wu, Pierangelo Metrangolo, Daniel Whitaker, Jade A. McCune, \\ and Oren A. Scherman*
}

Cite This: J. Am. Chem. Soc. 2020, 142, 7356-7361

Read Online

\section{ACCESS | Lill Metrics \& More | 国 Article Recommendations | St Supporting Information}

ABSTRACT: Phenyl-perfluorophenyl polar $-\pi$ interactions have been revisited for the design and fabrication of functional supramolecular systems. The relatively weak associative interactions $(\Delta G \approx-1.0 \mathrm{kcal} / \mathrm{mol})$ have limited their use in aqueous selfassembly to date. Herein, we propose a strategy to strengthen phenyl-perfluorophenyl polar $-\pi$ interactions by encapsulation within a synthetic host, thus increasing the binding affinity to $\Delta G=-15.5 \mathrm{kcal} / \mathrm{mol}$ upon formation of heteroternary complexes through social self-sorting. These heteroternary complexes were used as dynamic, yet strong, cross-linkers in the fabrication of supramolecular gels, which exhibited excellent viscoelasticity, stretchability, self-recovery, self-healing, and energy dissipation. This work unveils a general approach to exploit host-enhanced polar $-\pi$ interactions in the design of robust aqueous supramolecular systems.

$\mathrm{F}$ luorinated small molecules and macromolecules have been widely utilized in organic synthesis, ${ }^{1,2}$ polymer science, ${ }^{3}$ medicinal chemistry, ${ }^{4,5}$ drug delivery, ${ }^{6}$ liquid crystals, ${ }^{7}$ and supramolecular chemistry. ${ }^{8-11}$ Noncovalent interactions between aromatic molecules are important in both natural and artificial supramolecular systems. ${ }^{12}$ The introduction of electron-withdrawing fluorine substituents onto aromatic molecules serves to polarize their $\pi$-conjugated systems, enhancing $\pi-\pi$ stacking through electrostatic interactions. ${ }^{13,14}$ Phenyl-perfluorophenyl polar $-\pi$ interactions are an elegant example of electrostatic reinforcement. This interaction emerged from the first discovery of a 1:1 cocrystal of hexafluorobenzene and benzene in $1960^{15}$ (Figure 1a) followed by structural elucidation in 1992 by William et al. ${ }^{16}$ Coates and co-workers further developed phenyl-perfluorophenyl polar $-\pi$ interactions to align diyne molecules for photopolymerization in the crystalline state. ${ }^{17}$

While the perfluorophenyl group maintains preferential stacking selectivity with electron-rich arene groups, it also offers a position for functionalization. Thus, this interaction has been extensively adopted as a facile binding motif for crystal engineering, ${ }^{18-22}$ and protein/peptide folding, ${ }^{23-26}$ alongside small molecule and polymer self-assembly. ${ }^{27-30}$ Although significant progress has been made, the relatively low binding affinity of phenyl-perfluorophenyl polar $-\pi$ interactions in water $\left(\Delta G \approx-1.0 \mathrm{kcal} / \mathrm{mol}, K \approx 1.5 \mathrm{M}^{-1}\right)^{24}$ limits its widespread application.

Herein, we propose a host-guest strategy to mediate phenyl-perfluorophenyl polar $-\pi$ interactions, leading to a substantial increase in overall binding strength. There are several different host molecules capable of encapsulating multiple guests simultaneously. Among them, cucurbit[8]uril $(\mathrm{CB}[8])$ is one of the ideal options on account of its highenergy water release from its nanocavity upon guest incorporation, which can provide tremendous enthalpic contributions for stabilizing ternary host-guest complexes. ${ }^{31}$ $\mathrm{CB}[8]$-mediated ternary interactions often exhibit high binding a)

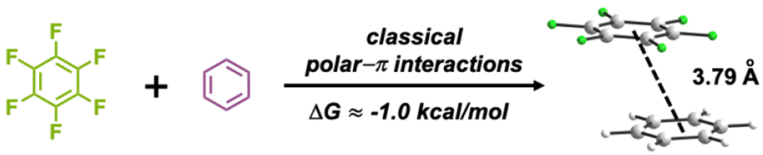

b)
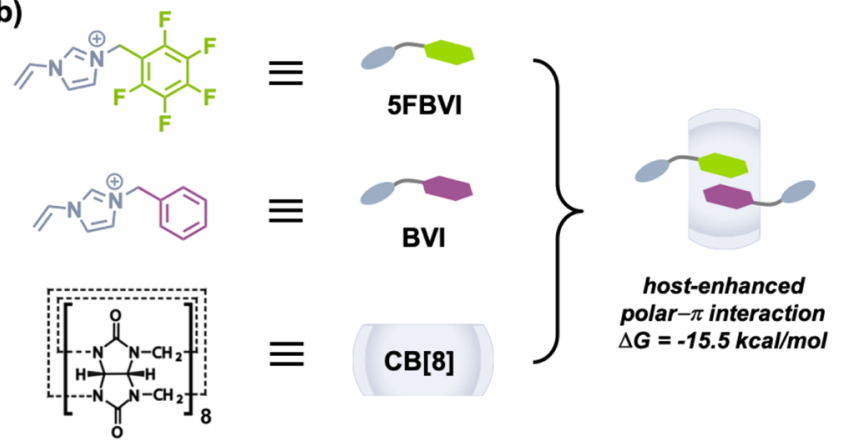

c)
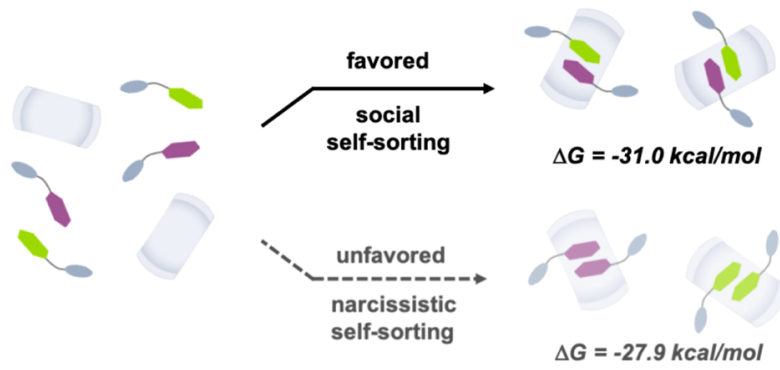

Figure 1. Schematic representation of (a) the first 1:1 cocrystal of hexafluorobenzene and benzene; (b) design concept of $\mathrm{CB}[8]$ mediated polar $-\pi$ interaction between perfluorophenyl (5FBVI) and phenyl (BVI) guests; (c) overview of thermodynamic pathways toward ternary complexation: social and narcissistic self-sorting. $\mathrm{Br}^{-}$ counterions omitted for clarity.

Received: March 5, 2020

Published: April 5, 2020 

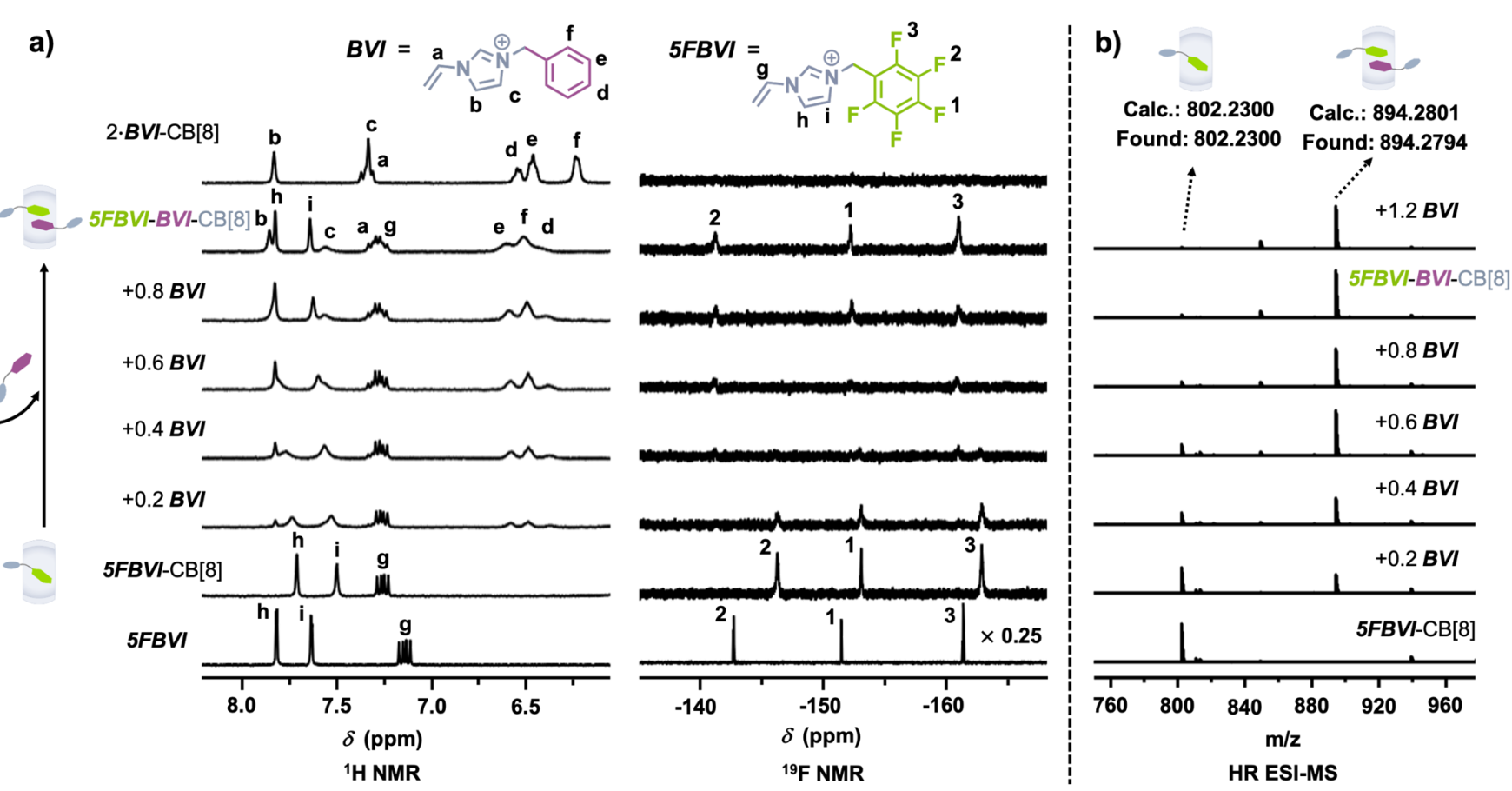

Figure 2. (a) ${ }^{1} \mathrm{H}$ and ${ }^{19} \mathrm{~F}$ NMR titrations $\left(\mathrm{D}_{2} \mathrm{O}, 298 \mathrm{~K}\right)$ and (b) HR ESI-MS titrations $\left(\mathrm{H}_{2} \mathrm{O}\right)$ of BVI (10.0 mM) into 5FBVI-CB[8] (1.0 mM). Br couterions omitted for clarity.

a)

positive cooperativity
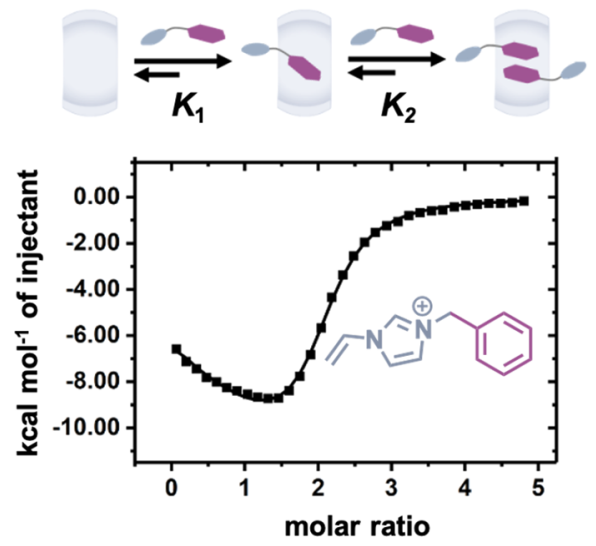

b) negative cooperativity

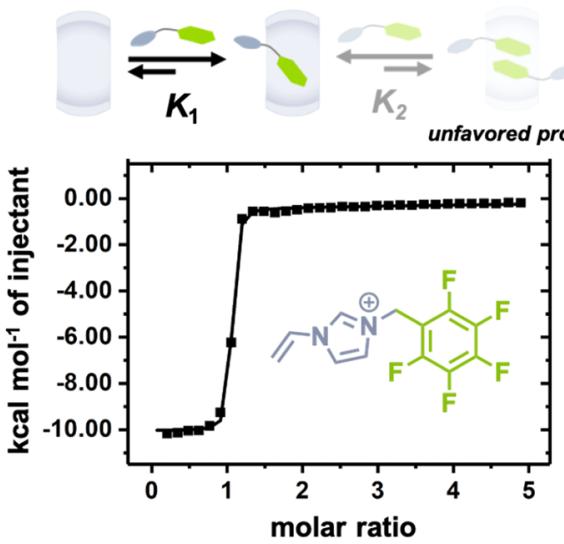

c)

secondary binding
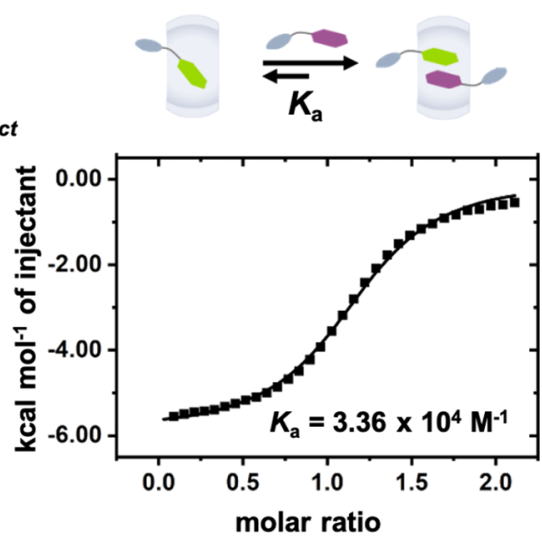

Figure 3. ITC curves $\left(\mathrm{H}_{2} \mathrm{O}, 298 \mathrm{~K}\right)$ of (a) BVI $(1.0 \mathrm{mM})$ into $\mathrm{CB}[8](0.05 \mathrm{mM})$; (b) 5FBVI (1.0 mM) into CB[8] (0.05 mM); and (c) BVI (5.0 $\mathrm{mM})$ into $5 \mathrm{FBVI}-\mathrm{CB}[8](0.5 \mathrm{mM}) . \mathrm{Br}^{-}$counterions omitted for clarity.

affinity $\left(\Delta G<-13.6 \mathrm{kcal} / \mathrm{mol}, K>10^{10} \mathrm{M}^{-2}\right) ;^{32}$ both homoternary and heteroternary interactions have been widely used as the driving force for fabrication of various aqueous supramolecular systems including supramolecular oligomers $^{33-35}$ and polymers, ${ }^{36-43}$ supramolecular hydrogels, ${ }^{44-47}$ supramolecular organic frameworks, ${ }^{48,49}$ protein/ peptide self-assemblies, ${ }^{50,51}$ and functional interfaces. ${ }^{52-54}$ Therefore, we posited that $\mathrm{CB}[8]$ could enhance phenylperfluorophenyl polar $-\pi$ interactions. Heteroternary complexation of singly charged guest molecules within $\mathrm{CB}[8]$ mediated by polar $-\pi$ interactions would represent a new class of $\mathrm{CB}[8]$ mediated heteroternary interaction, generating a promising and powerful noncovalent driving force for supramolecular selfassembly.

Two $N$-vinyl imidazolium guests were designed (Figure $1 \mathrm{~b}$ ), consisting of perfluorophenyl (5FBVI) and phenyl (BVI) groups. We envisioned that an equimolar mixture of 5FBVI,
BVI, and $\mathrm{CB}[8]$ should result exclusively in a $1: 1: 1$ heteroternary complex. Perfluorophenyl moieties should not form homoternary complexes on account of electrostatic repulsion, instead favoring secondary binding with an electronrich phenyl group. To study the influence of electrostatic repulsion, a series of guest molecules varying in fluorine substituents and cationic side groups were synthesized (Chart S1). We utilized the 5FBVI-BVI-CB[8] complex as a supramolecular cross-linker for fabricating robust transparent polyacrylamide networks exhibiting excellent mechanical properties and ionic conductivity.

Extensive ${ }^{1} \mathrm{H}$ and ${ }^{19} \mathrm{~F}$ NMR titrations were performed to study formation of the 5FBVI-BVI-CB[8] complex. Titration of BVI into an equimolar mixture of SFBVI and $\mathrm{CB}[8]$ resulted in the gradual appearance of the BVI phenyl protons at 6.39, 6.49, and $6.59 \mathrm{ppm}$, respectively (Figure 2a (left), S4). The phenyl proton peaks had similar chemical shifts to the 
homoternary complex $(2 \cdot \mathrm{BVI}-\mathrm{CB}[8])$ at $6.23,6.46$, and 6.54 ppm, suggesting the phenyl group in the 5FBVI-BVI-CB[8] complex is in a different chemical environment compared to 2 . BVI-CB $[8] .{ }^{19} \mathrm{~F}$ NMR (Figure 2a (right), S5) showed three peaks in the binary mixture of 5FBVI-CB $[8](-146.2,-153.0$, and $-162.8 \mathrm{ppm})$, which gradually disappeared while three new peaks at $-141.2,-152.2$, and $-160.9 \mathrm{ppm}$ appeared. Both titrations indicated formation of a new host-guest complex, 5FBVI-BVI-CB[8]. Additionally, a series of MS titration experiments were conducted. Titration of BVI into a $1: 1$ solution of 5FBVI-CB[8] yielded the heteroternary complex with an intense ion peak at $m / z=894.2794$, Figure 2 b, which gradually increased with a concomitant decrease of the binary complex at $m / z=802.2300$, confirming the $\mathrm{CB}[8]$-mediated polar $-\pi$ interaction.

Isothermal titration calorimetry (ITC) was employed to study the thermodynamic mechanism behind this new complexation. Titration of BVI and SFBVI into $\mathrm{CB}[8]$ led to two different cooperative binding curves. A clear transition at a 2:1 molar ratio was observed upon titration of BVI into $\mathrm{CB}[8]$ (Figure 3a), indicating formation of a homoternary complex. This binding curve is indicative of positive cooperativity, as reported previously. ${ }^{55}$ In contrast, titration of 5FBVI into $\mathrm{CB}[8]$ showed a sharp transition at 1 equiv (Figure $3 \mathrm{~b}$ ), indicating favored formation of a binary complex 5FBVI$\mathrm{CB}[8]\left(K_{1}=6.83 \times 10^{6} \mathrm{M}^{-1}\right.$, Table 1$)$. Incorporation of a

Table 1. Overview of Thermodynamic Data for Homoternary Complexations $\left(\mathrm{H}_{2} \mathrm{O}, 298 \mathrm{~K}\right)$

\begin{tabular}{lccc} 
& $K_{1}\left(10^{4} \mathrm{M}^{-1}\right)$ & $K_{2}\left(10^{4} \mathrm{M}^{-1}\right)$ & $\alpha$ \\
5FBVI & $683 \pm 187$ & $0.07 \pm 0.01$ & $0.0004 \pm 0.0001$ \\
4FBVI & $230 \pm 36$ & $33 \pm 6$ & $0.57 \pm 0.14$ \\
3FBVI & $95 \pm 28$ & $102 \pm 54$ & $4.27 \pm 2.61$ \\
2FBVI & $61 \pm 25$ & $69 \pm 43$ & $4.52 \pm 3.35$ \\
BVI & $44 \pm 5$ & $14 \pm 1$ & $1.29 \pm 0.16$ \\
\hline
\end{tabular}

second 5FBVI guest into the binary complex is significantly suppressed, exhibiting negative cooperativity. Free BVI was then titrated into the $1: 1$ binary complex of 5FBVI-CB[8] resulting in a secondary binding curve, $K_{\mathrm{a}}=3.36 \times 10^{4} \mathrm{M}^{-1}$ (Figure $3 \mathrm{c}$ ). The calculated overall binding constant of the heteroternary 5FBVI-BVI-CB[8] complex is $2.29 \times 10^{11} \mathrm{M}^{-2}$. Compared to the homoternary complex, $2 \cdot \mathrm{BVI}-\mathrm{CB}[8]$, this heteroternary complex exhibits higher binding strength on account of the additional electrostatic interactions between the phenyl and perfluorophenyl units.

Binding cooperativity plays a critical role in stabilizing the heteroternary 5FBVI-BVI-CB[8] complex; unexpectedly, a strong negative cooperativity with an interaction parameter ${ }^{55}$ $\alpha=0.0004$ was observed between 5FBVI and CB[8]. To gain insight into the importance of cooperativity, three $\mathrm{N}$-vinyl imidazolium guests (4FBVI, 3FBVI, 2FBVI) containing 2,3,5,6-tetrafluorophenyl, 3,4,5-trifluorophenyl, and 3,5-difluorophenyl groups, respectively, were synthesized. ITC indicated that $2: 1$ complexation with $\mathrm{CB}[8]$ was dramatically enhanced compared to SFBVI, Figure 4.

Table 1 reports key thermodynamic parameters; $\alpha$ values of 4FBVI, 3FBVI, and 2FBVI showed a steady increase, indicating a change from negative cooperativity for SFBVI and 4FBVI to positive cooperativity for 3FBVI and 2FBVI. These data indicate that weakening electrostatic repulsion between guests by decreasing the number of fluorine substituents leads to
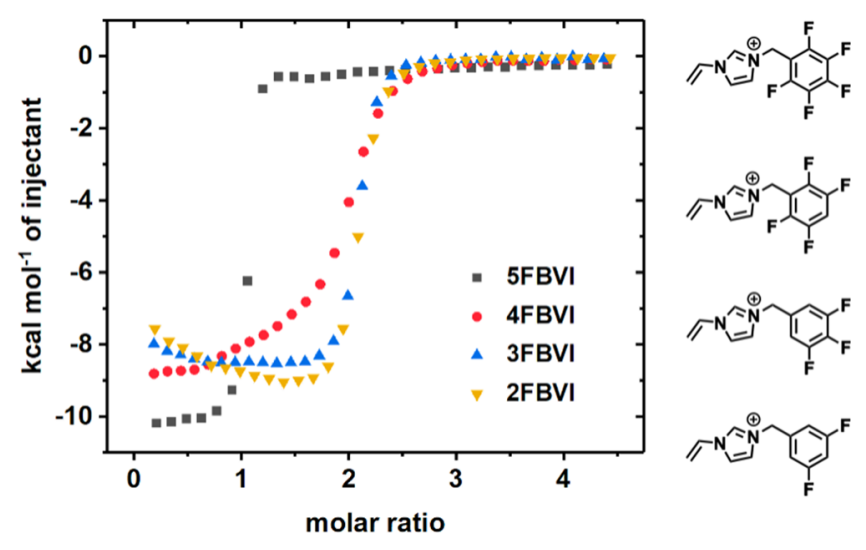

Figure 4. ITC plots $\left(\mathrm{H}_{2} \mathrm{O}, 298 \mathrm{~K}\right)$ of $\mathrm{CB}[8]$ homoternary complexation with 5FBVI, 4FBVI, 3FBVI, and 2FBVI. [Guest] = $1.0 \mathrm{mM},[\mathrm{CB}[8]]=0.05 \mathrm{mM}$.

favorable 2:1 homoternary complexation, thus changing the binding cooperativity from negative to positive. A previous report $^{38}$ of a heteroternary complex between tetrafluorophenyl, naphathalenyl, and $\mathrm{CB}[8]$ could support this effect; however, in this case additional long-range Coulombic and $\mathrm{CH} \cdots \mathrm{O}$ interactions are required to achieve binding as the complexation lacks strong negative cooperativity. In our study the 5FBVI-CB[8] complex is the only dominant $1: 1$ complex observed, Figure $3 \mathrm{~b}$.

To validate this point, and further extend the structural diversity for $\mathrm{CB}[8]$-mediated polar $-\pi$ interactions, three additional perfluorophenyl guests bearing $N$-methyl imidazolium, pyridinium, and trimethylammonium moieties were evaluated. The homoternary complexations of these three guests with $\mathrm{CB}[8]$ all exhibited strong negative cooperativity as expected (Figure S7), unambiguously demonstrating that the perfluorophenyl motif displays strong electrostatic repulsion within the $\mathrm{CB}[8]$ cavity, key to favoring the formation of a $1: 1$ complex.

The mechanism behind the formation of the heteroternary 5FBVI-BVI-CB [8] complex is attributed to social self-sorting, Figure 1c. Two complexation pathways exist for an equimolar mixture of 5FBVI, BVI, and CB[8]: social and narcissistic selfsorting. ${ }^{56}$ Thermodynamic data showed a substantial preference for social self-sorting with a difference in Gibbs free energy of $3.1 \mathrm{kcal} / \mathrm{mol}$ (Figure 1c), and a relative ratio between heteroternary and homoternary complex formation of nearly 200:1. Recently it was reported that a mixture of both social and narcissistic self-sorting is obtained with difluorophenyl guest motifs. ${ }^{57}$ In contrast, we are able to control the outcome of the self-sorting process, which results solely in the formation of the $\mathrm{CB}[8]$-mediated phenyl-perfluorophenyl complex through polar $-\pi$ interactions.

To demonstrate the utility of quantitative social self-sorting in $\mathrm{CB}[8]$-mediated polar $-\pi$ interactions, we employed the heteroternary complex 5FBVI-BVI-CB[8] as a supramolecular cross-linker to fabricate robust viscoelastic polyacrylamide networks. A mixture of $2.5 \mathrm{~mol} \%$ of 5FBVI-BVI-CB[8] crosslinker and $95 \mathrm{~mol} \%$ of acrylamide monomer with $0.003 \mathrm{~mol} \%$ photoinitiator was photopolymerized for $6 \mathrm{~h}(\lambda=350 \mathrm{~nm}$, Figure 5a). The resultant transparent gel could be readily stretched to 8-fold its original length demonstrating complete self-recovery within $3 \mathrm{~min}$. By reducing the ratio of supramolecular cross-linker, the storage and loss moduli of 

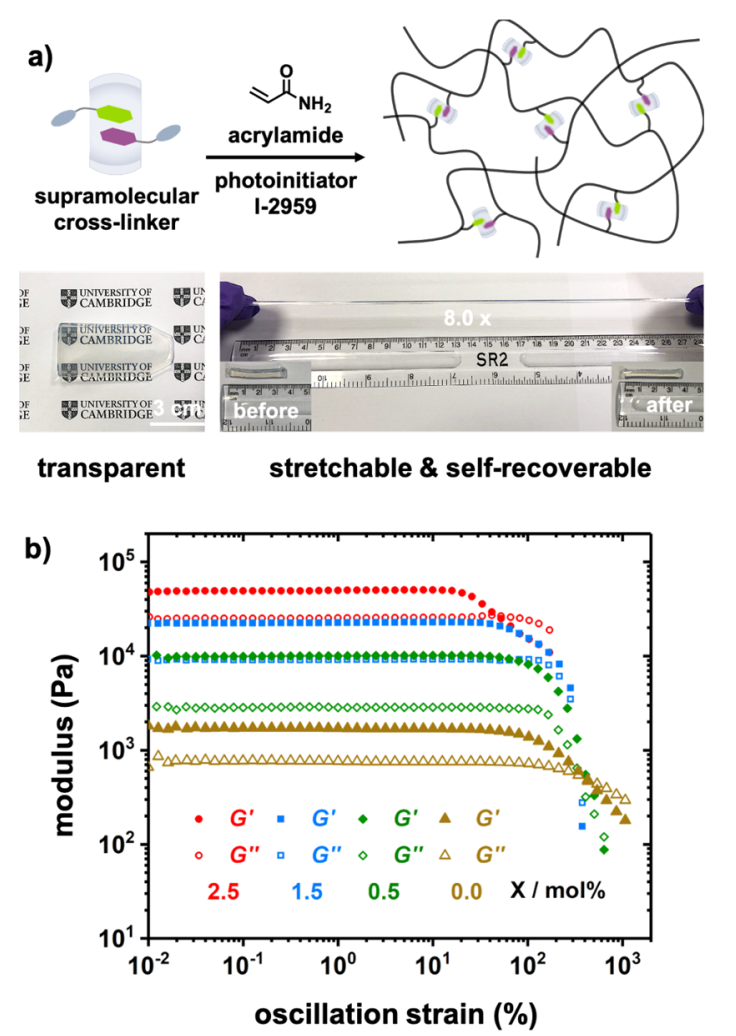

c)

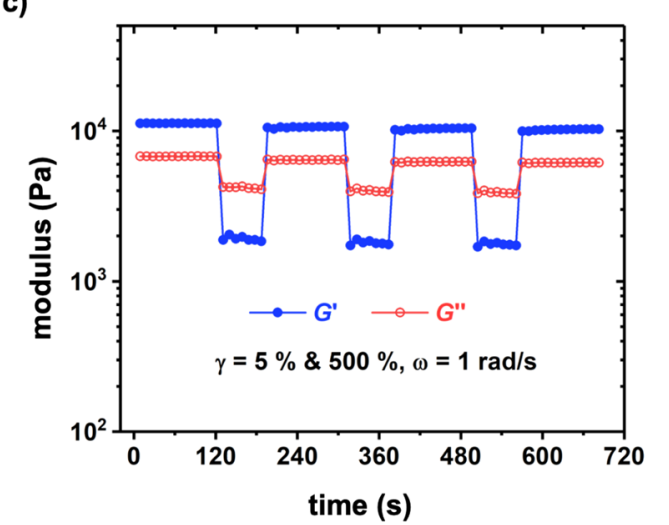

Figure 5. (a) Schematic representation of one-pot synthesis of 5FBVI-BVI-CB[8] cross-linked polyacrylamide network and photographs of obtained gel with $2.5 \mathrm{~mol} \%$ of 5FBVI-BVI-CB[8] crosslinker; (b) strain-sweep measurements of the formed gels with different cross-linker ratios $(\mathrm{X} / \mathrm{mol} \%)$; (c) continuous step-strain measurements of the gel with $2.5 \mathrm{~mol} \%$ cross-linker. Total $[$ monomer $]=2.0 \mathrm{M}$.

the obtained gels are readily tuned over a wide range, $10^{2}-10^{5} \mathrm{~Pa}$, Figures $5 \mathrm{~b}$ and $\mathrm{S} 12$.

The supramolecular gels (Figure $5 \mathrm{~b}$; green, blue, and red data sets) showed higher viscoelasticity with both storage and loss moduli 1-2 orders of magnitude above the functionalized copolymer gel without $\mathrm{CB}[8]$ present (yellow data set). Furthermore, when the 2.5 mol \% cross-linked gel was subjected to multiple step-strain cycles (5\% and 500\% strain at $1 \mathrm{rad} / \mathrm{s}$ ), a reversible sol-gel transition was observed, Figure 5c. This reversible behavior was replicated over a substantially longer time period $(>1 \mathrm{~h})$ without loss of material integrity, Figure S13. The resilience of the 5FBVI-BVI-CB[8] crosslinked gel toward step-strain measurements demonstrates the reversible and robust nature of the network and highlights its rapid self-healing properties.

Owing to the dynamic nature of $\mathrm{CB}[8]$-mediated cross-links, the transient polymeric networks dissipate energy upon application of a force, giving rise to excellent mechanical properties. ${ }^{46}$ To obtain the activation energy for local chain motion, a series of time-temperature superposition (TTS) experiments were carried out, quantifying the energydissipation capability of the 5FBVI-BVI-CB $[8]$ cross-linked gel (Figure S14). Fitting the shift parameters $\left(a_{T}, b_{T}\right)$ revealed the activation energy to be $18.0 \mathrm{kcal} / \mathrm{mol}$, comparable to the unfolding barrier of the $\mathrm{I} 27$ domain of the human muscle protein titin, which has an activation energy of $17.0 \mathrm{kcal} / \mathrm{mol}{ }^{58}$

On account of the charged 5FBVI and BVI guest moieties, we probed the ionic conductivity of the obtained material through incorporation within a series circuit connected to two LEDs, Figure S15. The high brightness of the LEDs connected to the gel in its original state illustrates its considerable ionic conductivity. Stretching the gel reduced its cross-sectional area, leading to an increase in resistance and an observed decrease in brightness of the LEDs. Removal of the stretching force returned the gel to its original length with concomitant increase in brightness over a 2 min period, demonstrating the gel's self-recovery properties.

In conclusion, we have successfully developed a new type of $\mathrm{CB}[8]$-mediated polar $-\pi$ interaction in aqueous media. Through host-guest complexation within $\mathrm{CB}[8]$, the binding affinity of phenyl-perfluorophenyl polar $-\pi$ interactions is significantly enhanced with an increase in $\Delta G$ from $-1.0 \mathrm{kcal} / \mathrm{mol}$ to $-15.5 \mathrm{kcal} / \mathrm{mol}$, assuring sole formation of the heteroternary complex upon social self-sorting. To demonstrate the utility of $\mathrm{CB}[8]$-mediated polar $-\pi$ interactions, we applied a model complex (5FBVI-BVI-CB $[8]$ ) as a supramolecular cross-linker in the fabrication of completely transparent polyacrylamide gel networks, which exhibited excellent viscoelasticity, stretchability, self-recovery, self-healing, and ionic conductivity. These materials are promising candidates for research in areas including structural biomaterials, flexible electronics, artificial muscles, soft robots, etc. We highly anticipate that this work will inspire renewed interest and research into the exploitation of polar $-\pi$ interactions as robust building blocks in supramolecular chemistry and materials science.

\section{ASSOCIATED CONTENT}

\section{Supporting Information}

The Supporting Information is available free of charge at https://pubs.acs.org/doi/10.1021/jacs.0c02275.

Materials and methods; molecular design and synthetic protocols; procedures for preparation of supramolecular polyacrylamide networks; study on $\mathrm{CB}[8]$-mediated polar $-\pi$ interactions by ${ }^{1} \mathrm{H}$ and ${ }^{19} \mathrm{~F}$ NMR, ESI-MS, and ITC; rheological characterization of supramolecular polyacrylamide networks; photographs for demonstration of ionic conductivity (PDF)

\section{AUTHOR INFORMATION}

\section{Corresponding Author}

Oren A. Scherman - Melville Laboratory for Polymer Synthesis, Department of Chemistry, University of Cambridge, Cambridge CB2 1EW, U.K.; (1) orcid.org/0000-0001-8032-7166; Email: oas23@cam.ac.uk 


\section{Authors}

Zehuan Huang - Melville Laboratory for Polymer Synthesis, Department of Chemistry, University of Cambridge, Cambridge CB2 1EW, U.K.

Xiaoyi Chen - Melville Laboratory for Polymer Synthesis, Department of Chemistry, University of Cambridge, Cambridge CB2 1EW, U.K.

Guanglu Wu - Melville Laboratory for Polymer Synthesis, Department of Chemistry, University of Cambridge, Cambridge CB2 1EW, U.K.; (1) orcid.org/0000-0002-9690-5992

Pierangelo Metrangolo - Department of Chemistry, Materials, and Chemical Engineering, "Giulio Natta", 20131 Milano, Italy; 이이.org/0000-0002-7945-099X

Daniel Whitaker - Melville Laboratory for Polymer Synthesis, Department of Chemistry, University of Cambridge, Cambridge CB2 $1 E W$, U.K.

Jade A. McCune - Melville Laboratory for Polymer Synthesis, Department of Chemistry, University of Cambridge, Cambridge CB2 1EW, U.K.

Complete contact information is available at:

https://pubs.acs.org/10.1021/jacs.0c02275

\section{Notes}

The authors declare no competing financial interest.

\section{ACKNOWLEDGMENTS}

Z.H. thanks Marie Skłodowska-Curie Fellowship (H2020MSCA-IF-2018, 845640). X.C. acknowledges CDT for financial support. D.W. thanks EPSRC $\mathrm{PhD}$ studentship (EP/R512461/1). G.W., J.A.M., and O.A.S. acknowledge an ERC consolidator grant (CAM-RIG, 726470) and EPSRC Programme Grant (NOtCH, EP/L027151/1) for financial support. We thank Dr. Robin Antrobus (Cambridge Institute for Medical Research) for help with MS.

\section{REFERENCES}

(1) O'Hagan, D. Understanding organofluorine chemistry. An introduction to the C-F bond. Chem. Soc. Rev. 2008, 37, 308-319.

(2) Furuya, T.; Kamlet, A. S.; Ritter, T. Catalysis for fluorination and trifluoromethylation. Nature 2011, 473, 470.

(3) Dhara, M. G.; Banerjee, S. Fluorinated high-performance polymers: poly (arylene ether) $\mathrm{s}$ and aromatic polyimides containing trifluoromethyl groups. Prog. Polym. Sci. 2010, 35, 1022-1077.

(4) Müller, K.; Faeh, C.; Diederich, F. Fluorine in pharmaceuticals: looking beyond intuition. Science 2007, 317, 1881-1886.

(5) Wang, J.; Sánchez-Roselló, M.; Aceña, J. L.; del Pozo, C.; Sorochinsky, A. E.; Fustero, S.; Soloshonok, V. A.; Liu, H. Fluorine in pharmaceutical industry: fluorine-containing drugs introduced to the market in the last decade (2001-2011). Chem. Rev. 2014, 114, 24322506.

(6) Krafft, M. P. Fluorocarbons and fluorinated amphiphiles in drug delivery and biomedical research. Adv. Drug Delivery Rev. 2001, 47, 209-228.

(7) Hird, M. Fluorinated liquid crystals-properties and applications. Chem. Soc. Rev. 2007, 36, 2070-2095.

(8) Gale, P. A.; Tong, C. C.; Haynes, C. J.; Adeosun, O.; Gross, D. E.; Karnas, E.; Sedenberg, E. M.; Quesada, R.; Sessler, J. L. Octafluorocalix [4] pyrrole: a chloride/bicarbonate antiport agent. J. Am. Chem. Soc. 2010, 132, 3240-3241.

(9) Meazza, L.; Foster, J. A.; Fucke, K.; Metrangolo, P.; Resnati, G.; Steed, J. W. Halogen-bonding-triggered supramolecular gel formation. Nat. Chem. 2013, 5, 42-47.

(10) Takezawa, H.; Murase, T.; Resnati, G.; Metrangolo, P.; Fujita, M. Recognition of polyfluorinated compounds through selfaggregation in a cavity. J. Am. Chem. Soc. 2014, 136, 1786-1788.
(11) Zhang, X.; Hao, X.; Liu, L.; Pham, A.-T.; López-Andarias, J.; Frontera, A.; Sakai, N.; Matile, S. Primary Anion- $\pi$ Catalysis and Autocatalysis. J. Am. Chem. Soc. 2018, 140, 17867-17871.

(12) Meyer, E. A.; Castellano, R. K.; Diederich, F. Interactions with aromatic rings in chemical and biological recognition. Angew. Chem., Int. Ed. 2003, 42, 1210-1250.

(13) Hunter, C. A.; Sanders, J. K. The nature of $\pi-\pi$ interactions. J. Am. Chem. Soc. 1990, 112, 5525-5534.

(14) Martinez, C. R.; Iverson, B. L. Rethinking the term " $\pi$-stacking". Chem. Sci. 2012, 3, 2191-2201.

(15) Patrick, C.; Prosser, G. A molecular complex of benzene and hexafluorobenzene. Nature 1960, 187, 1021.

(16) Williams, J. H.; Cockcroft, J. K.; Fitch, A. N. Structure of the lowest temperature phase of the solid benzene-hexafluorobenzene adduct. Angew. Chem., Int. Ed. Engl. 1992, 31, 1655-1657.

(17) Coates, G. W.; Dunn, A. R.; Henling, L. M.; Dougherty, D. A.; Grubbs, R. H. Phenyl-perfluorophenyl stacking interactions: A new strategy for supermolecule construction. Angew. Chem., Int. Ed. Engl. 1997, 36, 248-251.

(18) Ponzini, F.; Zagha, R.; Hardcastle, K.; Siegel, J. S. Phenyl/ pentafluorophenyl interactions and the generation of ordered mixed crystals: sym-triphenethynylbenzene and sym-tris (perfluorophenethynyl) benzene. Angew. Chem., Int. Ed. 2000, 39, 2323-2325.

(19) Watt, S. W.; Dai, C.; Scott, A. J.; Burke, J. M.; Thomas, R. L.; Collings, J. C.; Viney, C.; Clegg, W.; Marder, T. B. Structure and phase behavior of a 2:1 complex between arene-and fluoroarene-based conjugated rigid rods. Angew. Chem., Int. Ed. 2004, 43, 3061-3063.

(20) Rasmusson, T.; Martyn, L. J. P.; Chen, G.; Lough, A.; Oh, M.; Yudin, A. K. Aromatic fluorine as a versatile control element for the construction of molecules with helical chirality. Angew. Chem., Int. Ed. 2008, 47, 7009-7012.

(21) Xu, R.; Schweizer, W. B.; Frauenrath, H. Soluble poly (diacetylene) $\mathrm{s}$ using the perfluorophenyl- phenyl motif as a supramolecular synthon. J. Am. Chem. Soc. 2008, 130, 11437-11445.

(22) Pawle, R.; Haas, T.; Müller, P.; Thomas, S., III Twisting and piezochromism of phenylene-ethynylenes with aromatic interactions between side chains and main chains. Chem. Sci. 2014, 5, 4184-4188.

(23) Cejas, M. A.; Kinney, W. A.; Chen, C.; Leo, G. C.; Tounge, B. A.; Vinter, J. G.; Joshi, P. P.; Maryanoff, B. E. Collagen-related peptides: Self-assembly of short, single strands into a functional biomaterial of micrometer scale. J. Am. Chem. Soc. 2007, 129, 22022203.

(24) Zheng, H.; Gao, J. Highly specific heterodimerization mediated by quadrupole interactions. Angew. Chem., Int. Ed. 2010, 49, 86358639.

(25) Pace, C. J.; Zheng, H.; Mylvaganam, R.; Kim, D.; Gao, J. Stacked fluoroaromatics as supramolecular synthons for programming protein dimerization specificity. Angew. Chem., Int. Ed. 2012, 51, 103107.

(26) Zhang, C.; Welborn, M.; Zhu, T.; Yang, N. J.; Santos, M. S.; Van Voorhis, T.; Pentelute, B. L. $\pi$-Clamp-mediated cysteine conjugation. Nat. Chem. 2016, 8, 120.

(27) Kishikawa, K.; Oda, K.; Aikyo, S.; Kohmoto, S. Columnar superstructures of non-disc-shaped molecules generated by areneperfluoroarene face-to-face interactions. Angew. Chem., Int. Ed. 2007, 46, 764-768.

(28) Lu, J.; Ten Brummelhuis, N.; Weck, M. Intramolecular folding of triblock copolymers via quadrupole interactions between poly (styrene) and poly (pentafluorostyrene) blocks. Chem. Commun. 2014, 50, 6225-6227.

(29) Sharber, S. A.; Baral, R. N.; Frausto, F.; Haas, T. E.; Müller, P.; Thomas, S. W., III Substituent effects that control conjugated oligomer conformation through non-covalent interactions. J. Am. Chem. Soc. 2017, 139, 5164-5174.

(30) Elacqua, E.; Geberth, G. T.; Bout, D. A. V.; Weck, M. Synthesis and folding behaviour of poly ( $\mathrm{p}$-phenylene vinylene)-based $\beta$-sheet polychromophores. Chem. Sci. 2019, 10, 2144-2152.

(31) Biedermann, F.; Vendruscolo, M.; Scherman, O. A.; De Simone, A.; Nau, W. M. Cucurbit[8]uril and blue-box: high-energy 
water release overwhelms electrostatic interactions. J. Am. Chem. Soc. 2013, 135, 14879-14888.

(32) Barrow, S. J.; Kasera, S.; Rowland, M. J.; del Barrio, J.; Scherman, O. A. Cucurbituril-based molecular recognition. Chem. Rev. 2015, 115, 12320-12406.

(33) Ko, Y. H.; Kim, K.; Kang, J.-K.; Chun, H.; Lee, J. W.; Sakamoto, S.; Yamaguchi, K.; Fettinger, J. C.; Kim, K. Designed self-assembly of molecular necklaces using host-stabilized charge-transfer interactions. J. Am. Chem. Soc. 2004, 126, 1932-1933.

(34) Wu, G.; Olesińska, M.; Wu, Y.; Matak-Vinkovic, D.; Scherman, O. A. Mining 2:2 complexes from 1:1 stoichiometry: formation of cucurbit [8] uril-diarylviologen quaternary complexes favored by electron-donating substituents. J. Am. Chem. Soc. 2017, 139, 32023208.

(35) Tang, B.; Li, W.-L.; Chang, Y.; Yuan, B.; Wu, Y.; Zhang, M.-T.; Xu, J.-F.; Li, J.; Zhang, X. A Supramolecular Radical Dimer: HighEfficiency NIR-II Photothermal Conversion and Therapy. Angew. Chem., Int. Ed. 2019, 58, 15526-15531.

(36) Liu, Y.; Yu, Y.; Gao, J.; Wang, Z.; Zhang, X. Water-soluble supramolecular polymerization driven by multiple host-stabilized charge-transfer interactions. Angew. Chem., Int. Ed. 2010, 49, 65766579.

(37) Huang, Z.; Yang, L.; Liu, Y.; Wang, Z.; Scherman, O. A.; Zhang, $X$. Supramolecular polymerization promoted and controlled through self-sorting. Angew. Chem., Int. Ed. 2014, 53, 5351-5355.

(38) Joseph, R.; Nkrumah, A.; Clark, R. J.; Masson, E. Stabilization of Cucurbituril/Guest Assemblies via Long-Range Coulombic and CH‥ O Interactions. J. Am. Chem. Soc. 2014, 136, 6602-6607.

(39) Ni, X.-L.; Chen, S.; Yang, Y.; Tao, Z. Facile cucurbit [8] urilbased supramolecular approach to fabricate tunable luminescent materials in aqueous solution. J. Am. Chem. Soc. 2016, 138, 61776183.

(40) Samanta, S. K.; Moncelet, D.; Briken, V.; Isaacs, L. Metalorganic polyhedron capped with cucurbit $[8]$ uril delivers doxorubicin to cancer cells. J. Am. Chem. Soc. 2016, 138, 14488-14496.

(41) Raeisi, M.; Kotturi, K.; del Valle, I.; Schulz, J.; Dornblut, P.; Masson, E. Sequence-Specific Self-Assembly of Positive and Negative Monomers with Cucurbit [8] uril Linkers. J. Am. Chem. Soc. 2018, 140, 3371-3377.

(42) Wu, H.; Chen, Y.; Dai, X.; Li, P.; Stoddart, J. F.; Liu, Y. In-situ photoconversion of multicolor luminescence and pure white light emission based on carbon dot-supported supramolecular assembly. J. Am. Chem. Soc. 2019, 141, 6583-6591.

(43) Yin, Z.; Song, G.; Jiao, Y.; Zheng, P.; Xu, J.-F.; Zhang, X. Dissipative supramolecular polymerization powered by light. CCS Chem. 2019, 1, 335-342.

(44) Appel, E. A.; Biedermann, F.; Rauwald, U.; Jones, S. T.; Zayed, J. M.; Scherman, O. A. Supramolecular cross-linked networks via hostguest complexation with cucurbit [8] uril. J. Am. Chem. Soc. 2010, 132, 14251-14260.

(45) Appel, E. A.; Loh, X. J.; Jones, S. T.; Biedermann, F.; Dreiss, C. A.; Scherman, O. A. Ultrahigh-water-content supramolecular hydrogels exhibiting multistimuli responsiveness. J. Am. Chem. Soc. 2012, 134, 11767-11773.

(46) Liu, J.; Tan, C. S. Y.; Yu, Z.; Li, N.; Abell, C.; Scherman, O. A. Tough supramolecular polymer networks with extreme stretchability and fast room-temperature self-healing. Adv. Mater. 2017, 29, 1605325.

(47) Xu, W.; Song, Q.; Xu, J.-F.; Serpe, M. J.; Zhang, X. Supramolecular hydrogels fabricated from supramonomers: a novel wound dressing material. ACS Appl. Mater. Interfaces 2017, 9, 1136811372.

(48) Zhang, K.-D.; Tian, J.; Hanifi, D.; Zhang, Y.; Sue, A. C.-H.; Zhou, T.-Y.; Zhang, L.; Zhao, X.; Liu, Y.; Li, Z.-T. Toward a singlelayer two-dimensional honeycomb supramolecular organic framework in water. J. Am. Chem. Soc. 2013, 135, 17913-17918.

(49) Li, Y.; Dong, Y.; Miao, X.; Ren, Y.; Zhang, B.; Wang, P.; Yu, Y.; Li, B.; Isaacs, L.; Cao, L. Shape-controllable and fluorescent supramolecular organic frameworks through aqueous host-guest complexation. Angew. Chem., Int. Ed. 2018, 57, 729-733.

(50) de Vink, P. J.; Briels, J. M.; Schrader, T.; Milroy, L.-G.; Brunsveld, L.; Ottmann, C. A Binary bivalent supramolecular assembly platform based on cucurbit[8] uril and dimeric adapter protein 14-3-3. Angew. Chem., Int. Ed. 2017, 56, 8998-9002.

(51) Hirani, Z.; Taylor, H. F.; Babcock, E. F.; Bockus, A. T.; Varnado, C. D., Jr; Bielawski, C. W.; Urbach, A. R. Molecular recognition of methionine-terminated peptides by cucurbit[8]uril. J. Am. Chem. Soc. 2018, 140, 12263-12269.

(52) Sankaran, S.; Kiren, M. C.; Jonkheijm, P. Incorporating bacteria as a living component in supramolecular self-assembled monolayers through dynamic nanoscale interactions. ACS Nano 2015, 9, 35793586.

(53) Liu, J.; Scherman, O. A. Cucurbit [n] uril supramolecular hydrogel networks as tough and healable adhesives. Adv. Funct. Mater. 2018, 28, 1800848.

(54) Liu, J.; Tan, C. S. Y.; Scherman, O. A. Dynamic interfacial adhesion through cucurbit[n]uril molecular recognition. Angew. Chem., Int. Ed. 2018, 57, 8854-8858.

(55) Huang, Z.; Qin, K.; Deng, G.; Wu, G.; Bai, Y.; Xu, J.-F.; Wang, Z.; Yu, Z.; Scherman, O. A.; Zhang, X. Supramolecular chemistry of cucurbiturils: tuning cooperativity with multiple noncovalent interactions from positive to negative. Langmuir 2016, 32, 1235212360.

(56) Safont-Sempere, M. M.; Fernández, G.; Würthner, F. Selfsorting phenomena in complex supramolecular systems. Chem. Rev. 2011, 111, 5784-5814.

(57) Barbero, H.; Thompson, N. A.; Masson, E. Dual layer" selfsorting with Cucurbiturils. J. Am. Chem. Soc. 2020, 142, 867-873.

(58) Muddassir, M.; Manna, B.; Singh, P.; Singh, S.; Kumar, R.; Ghosh, A.; Sharma, D. Single-molecule force-unfolding of titin I27 reveals a correlation between the size of the surrounding anions and its mechanical stability. Chem. Commun. 2018, 54, 9635-9638. 